\title{
Growth pattern of uterine leiomyoma along pregnancy
}

Henry Hillel Chill ${ }^{1 *+}$ (D) Gilad Karavani ${ }^{1+}$, Talya Rachmani ${ }^{1}$, Uri Dior ${ }^{1}$, Ofer Tadmor ${ }^{2}$ and Asher Shushan ${ }^{1,2}$

\begin{abstract}
Background: Uterine leiomyomas are often discovered during early pregnancy and in most cases will have no effect on pregnancy outcomes. However, in rare cases uterine leiomyomas may lead to obstetric complications. The aim of the study was to evaluate rate of uterine leiomyoma growth in the 3 trimesters of pregnancy.

Methods: We conducted a retrospective cohort study. Included were women who were diagnosed with uterine leiomyoma during pregnancy and had at least two sonographic measurements in different trimesters. Data regarding leiomyoma growth, recorded by ultrasound examination, during 1st 2nd and 3rd trimesters were collected from electronic patient records.

Results: Two-hundred forty-eight uterine leiomyomas were included in the study. Leiomyoma area increased substantially in size between the 1 st and 2 nd trimesters $(54.5 \% \pm 75.9 \%, p=.007)$ and to a lesser degree between the 2nd and 3rd trimesters $(17.9 \% \pm 59.7 \%$, NS). Evaluation of the change in size throughout the pregnancy - between 1st and 3rd trimesters revealed a significant increase of $95.9 \% \pm 191.3 \%(p<.001)$. There was no significant growth of the leiomyomas between the 2nd and 3rd trimesters.

Conclusions: Uterine leiomyomas tend to grow substantially during the 1st trimester of pregnancy. This trend is attenuated later with minimal growth towards the end of gestation.
\end{abstract}

Keywords: Uterine leiomyoma, Uterine fibroid, Pregnancy, Ultrasound, Complicated pregnancy

\section{Background}

Uterine leiomyomas have challenged the scientific community for the past two centuries and yet the pathogenesis of uterine leiomyoma growth is still unclear. Estrogen and progesterone have been implicated as important instigators of leiomyoma growth together with cytokines, growth factors, chemokines and other regulatory proteins [1-4]. Recent studies have pointed towards Human Chorionic Gonadotropin (HCG) as a major contributor towards Leiomyoma growth $[5,6]$.

Uterine leiomyomas are often an incidental finding discovered during an ultrasound (US) examination performed in early pregnancy [7]. In pregnancy, their prevalence is estimated at $2.7-10.7 \%$ with most women being asymptomatic with uneventful pregnancies [7-10]. However, leiomyomas can manifest during pregnancy with pain and discomfort

\footnotetext{
* Correspondence: henchill@gmail.com; Henchill@gmail.com

${ }^{+}$Henry Hillel Chill and Gilad Karavani contributed equally to this work.

${ }^{1}$ Department of Obstetrics and Gynecology, Hadassah Hebrew University

Medical Center, Ein-Kerem, PO Box 12000, 91120 Jerusalem, Israel

Full list of author information is available at the end of the article
}

due to torsion or degeneration. They have also been associated with early pregnancy failure as well as with obstetric complications such as preterm labor, abnormal fetal lie and presentation, placental abruption, post-partum hemorrhage and retained placenta [11].

The purpose of our study was to describe rate of leiomyoma growth in the 3 trimesters of pregnancy.

\section{Methods}

We conducted a retrospective cohort study. Data were collected between January 2012 and December 2016 from the database of one of the main Israeli health maintenance organizations (Maccabi Health Services). The study was approved by the institutional ethical review board and no informed consent was required.

Included in the study were women over the age of 18 who underwent routine US exam during pregnancy and who were diagnosed with at least one uterine leiomyoma. All women had at least two measurements

(c) The Author(s). 2019 Open Access This article is distributed under the terms of the Creative Commons Attribution 4.0 International License (http://creativecommons.org/licenses/by/4.0/), which permits unrestricted use, distribution, and 
during two different trimesters. Measurements included at least a bi-dimensional estimation of the leiomyoma size $(\mathrm{mm})$. Excluded were women who failed to meet all inclusion criteria.

First trimester was defined up to $11+6$ weeks gestation, 2nd trimester between 12 and $23+6$ weeks gestation and the 3 rd trimester between $24+0$ weeks and date of delivery.

Demographic data collected included maternal age, parity, body mass index (BMI) and gestational age during the US exams. Leiomyoma characteristics included length and width in $(\mathrm{mm})$ and area (length $\mathrm{X}$ width). Number of leiomyomas, location (right, left, anterior, posterior, fundal and cervical) and topographic site (sub-serosal, intramural and sub-mucosal) were also evaluated. In women who had multiple leiomyomas follow up was performed individually for each leiomyoma according to its original location.

The primary outcome was uterine leiomyoma growth pattern along pregnancy. Secondary outcomes included correlation between different parameters investigated and the percent of weekly or overall change in area of leiomyomas between trimesters.

Evaluation of uterine leiomyoma size included the following comparisons: (1) Size in the 1st trimester compared to 3rd trimester; (2) 1st trimester compared to 2nd trimester; (3) 2nd trimester compared to 3rd trimester and (4) 3rd trimester compared to 6 weeks postdelivery (available only for a subset of patients).

US exams were performed by either US technicians or physicians with expertise in performing such diagnostic studies.

Leiomyoma size was approximated by calculating the area of each tumor (length in $\mathrm{mm} \mathrm{X}$ width in $\mathrm{mm})$. Change in leiomyoma area was calculated as the difference between the latest area measurement and the initial area measurement, both in $\mathrm{mm}^{2}$. The growth rate per week, presented as increase in percent, was calculated using the following formula: [100 X (Change in area) / (Initial area)] / (Interval in weeks between measurements) [7].

\section{Statistical analysis}

To assess the change between the two time points paired $t$ test, the one sample t-test and the Wilcoxon signed ranks test were applied. The Pearson Correlation Coefficient and the Spearman Non Parametric Correlation Coefficient were used to assess the strength of the association between two quantitative variables.

Comparisons of quantitative variables between 2 and 3 independent groups were carried-out using the Mann-Whitney non parametric test and the nonparametric Kruskal-Wallis test, respectively. All tests applied were two tailed and a $P$-value of 0.05 or less was considered statistically significant.

\section{Results}

During the study period, 377 patients with 458 uterine leiomyomas were evaluated sonographically. After applying our inclusion criteria, 196 patients with an overall number of 248 leiomyomas were included in the analysis.

Of the 248 leiomyomas, 173 were assessed at least once in two different trimesters of gestation and 75 leiomyomas were evaluated at least once in each of three trimesters of gestation. A flow chart depicting the study population is presented in Fig. 1.

Basic characteristics of the study population and leiomyoma location are presented in Table 1. Table 2 shows the change in area of leiomyomas between the 1st and 2nd trimesters and 2nd and 3rd trimesters. The majority of leiomyomas increased in size between the 1st and 2nd trimesters $(p<0.01)$. Change in growth of leiomyomas between the 2nd and 3rd trimester did not reach statistical significance. Initial leiomyoma size was not found to have an effect on growth during pregnancy.

Seventy-five leiomyomas were measured at least once during each of the three trimesters of gestation. In this subgroup, the overall increase in leiomyoma area between 1 st and 2nd trimesters was $60.62 \% \pm 58.37 \%$ $(p<.001)$ and the overall increase in leiomyoma area between 2nd and 3rd trimesters was 8.2943.57 $\pm \%$ $(p<.001)$. The percent of change in area throughout the 1 st and 3rd trimesters was $74.13 \pm 109.59 \%(p<.001)$.

A further analysis, assessing change in area per week, has shown a weekly increase in size of leiomyomas of $6.90 \% \pm$ 6.12\% $(p<.001)$ between 1st and 2nd trimesters and a $3.10 \% \pm 0.39 \%$ between 2 nd and 3rd trimesters $(p<.05)$.

All 54 leiomyomas measured in the 3rd trimester and 6 weeks post delivery decreased significantly. The average reduction in size was $44.25 \% \pm 36.05 \%(p<.05)$.

We further analysed the change in size between gestational trimesters per specific location (posterior, anterior, right, left, fundal, cervical) and topographic site (sub-serosal, intramural, and sub-mucosal). A significant increase in leiomyoma area was demonstrated between the 1st and 2nd trimesters for location and topographic site. A significant increase in leiomyoma area was also demonstrated between the 2 nd and $3 \mathrm{rd}$ trimesters for the anterior and right leiomyomas and in sub-serosal and intramural topographic sites. Statistical significance was lost when growth was analyzed per week.

Cervical leiomyomas assessed in the different trimesters showed a significant weekly and overall increase in size between the 1 st and 2 nd trimesters $(7.63 \pm 6.28 \%$ and $94.22 \pm 70.25 \%$, respectively; $p<.05$ ). However, this trend subsided between the 2nd and 3rd trimesters. 


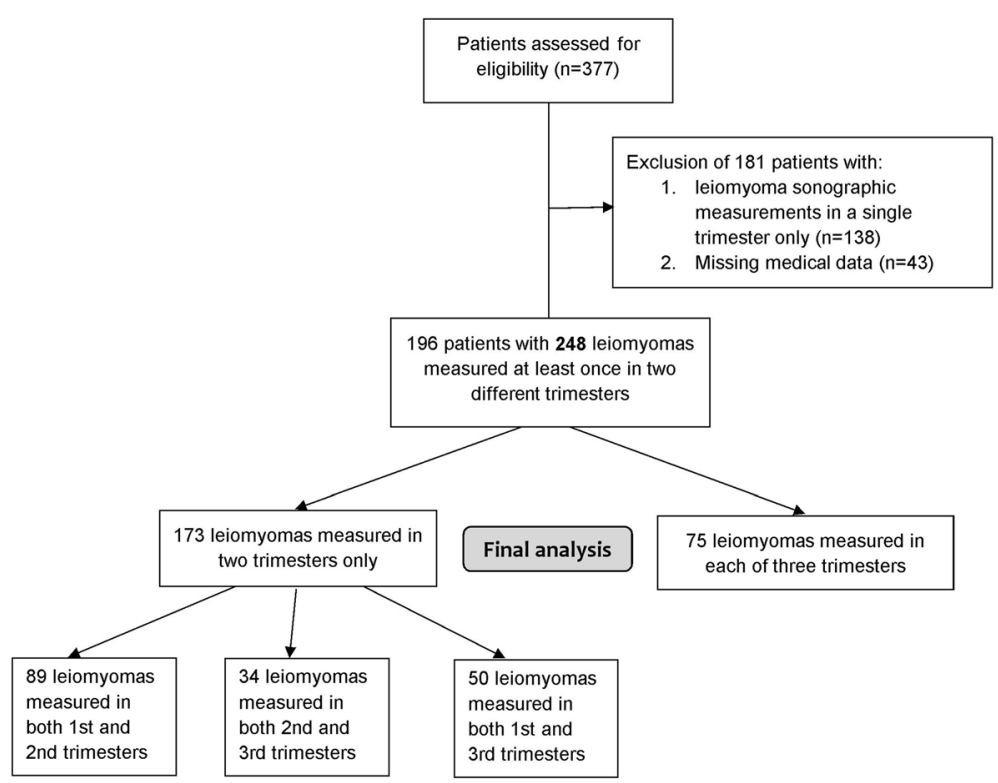

Fig. 1 Flow chart of patient inclusion for final analysis

No correlation was found between age, gravidity, parity, BMI, number of leiomyomas, location and topographic site of leiomyomas and the percent of weekly or overall change in area of leiomyomas between trimesters (Table 3 ).

Table 1 Basic characteristics of the study population (196 women with an overall number of 248 leiomyoma)

\begin{tabular}{ll}
\hline Parameter & $\mathrm{n}(\%)$ or mean \pm SD \\
\hline Age (years) (Range) & $35.90 \pm 5.07(20-53)$ \\
Body Mass Index (BMI) (Range) & $28.33 \pm 5.91$ (16-47) \\
Gravidity & $2.52 \pm 1.93$ \\
Parity & $0.98 \pm 1.45$ \\
Multiple gestation pregnancy & $16(6.5 \%)$ \\
Leiomyoma location & \\
$\quad$ Posterior & $75(30.2 \%)$ \\
$\quad$ Anterior & $79(31.9 \%)$ \\
$\quad$ Right & $34(13.7 \%)$ \\
Left & $22(8.9 \%)$ \\
Fundal & $23(9.3 \%)$ \\
Cervical & $15(6.1 \%)$ \\
Leiomyoma topographic location & \\
Subserosal & \\
Intramural & $88(51.8 \%)$ \\
Submucosal & $68(40.0 \%)$ \\
Leiomyoma length pre-conception (mm) & $14(8.2 \%)$ \\
Leiomyoma width pre-conception (mm) & $44.91 \pm 20.07$ \\
\hline
\end{tabular}

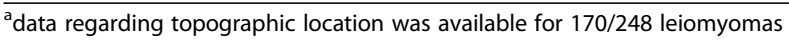

\section{Discussion}

Uterine leiomyomas are a relatively common finding during pregnancy. While often asymptomatic, leiomyomas have the potential of becoming clinically important during pregnancy, depending on size and location [7]. Over the years an attempt has been made to describe the natural history of uterine leiomyomas during pregnancy. Previous studies have presented equivocal data with some studies describing increase in leiomyoma size during pregnancy while others report no change or decrease in size [7, 12-15].

In our study we found that uterine leiomyomas have a distinct growth pattern during pregnancy. We have shown that while leiomyomas increase in size during pregnancy, their growth pattern is differential with a substantial increase in size between the 1st and 2nd trimesters that is later attenuated.

Our results correlate partially with data published in previous studies. In their systematic review of the literature Vitagliano et al. reported on distinct leiomyoma growth between the 1st and 2nd trimesters with contradictory evidence with respect to 3rd trimester growth [12]. De Vivo et al. showed an increase in leiomyoma size between the 1st and 2nd trimesters as well as between the 2nd and 3rd trimesters [7]. In another series, Aharoni et al. presented leiomyomas to be mostly unchanged during pregnancy (59\%). In cases where increase in size was noted (22\%) growth percentage was minor (25\%) [13]. In a series of 137 leiomyomas in 72 women Neiger et al. found similar results with no change in leiomyoma size during pregnancy [14]. 
Table 2 Modifications in leiomyoma area throughout gestation in patients with at least one measurement in two different trimesters

\begin{tabular}{llll}
\hline & 1st vs. 2nd trimester & 2nd vs. 3rd trimester & 1st vs. 3rd trimester \\
\hline N of leiomyomas & 164 & 109 & 125 \\
Increase in size & $130(79.3 \%)$ & $55(50.4 \%)$ & $100(80.0 \%)$ \\
Decrease in size & $33(20.1 \%)$ & $53(48.6 \%)$ & $24(19.2 \%)$ \\
No change & $1(0.6 \%)$ & $1(9.2 \%)$ & $1(0.8 \%)$ \\
Mean change (\%) $_{P \text { value }^{\text {a }}}$ & $54.54 \pm 75.87 \%$ & $17.88 \pm 59.71 \%$ & $95.90 \pm 191.31 \%$ \\
\hline
\end{tabular}

Data presented as $\mathrm{n}(\%)$ or mean $\pm \mathrm{SD}$

${ }^{\text {a }}$ Statistical significance of the mean change (\%) in size between trimesters

In our study the initial leiomyoma size was not found to have an effect on growth during pregnancy. Contrary to our findings Lev-Toaff et al. followed 113 pregnant women with uterine leiomyoma while taking into account their original size. They showed that small leiomyomas grew during the 1st and 2nd trimesters but reduced in size during the 3rd trimester as opposed to large leiomyomas which grew during the first trimester but shrunk during the 2nd and 3rd trimesters [15].

Previous studies showed a possible association between leiomyoma growth and multi-parity, pre-pregnancy body mass index and maternal age [7]. Multivariate analysis performed in this study did not find any linkage between leiomyoma growth and any of the parameters investigated.

Full understanding of the pathogenesis of uterine leiomyoma is still lacking but clearly sex steroid hormones play a major role in their growth process [2, 3]. However, estrogen and progesterone are unlikely to be the sole actors involved. Estrogen and progesterone serum levels peak during the 3rd trimester of pregnancy while in most studies the major increase in leiomyoma size occurs in the 1st trimester [7]. This has led researchers to search for other proteins and hormones that might affect leiomyoma growth during pregnancy.

A large body of evidence has accumulated in favor of HCG as a possible stimulator of leiomyoma growth $[5,6]$. This stimulatory effect seems to be mediated by the leuteinizing hormone/HCG receptor complex.
This complex was shown to be expressed in myometrium as well as in leiomyomas in animals and humans [16]. It is postulated that these receptors are sensitive to exponential rise in HCG levels. As pregnancy advances down regulation of these receptors stunts leiomyoma growth leading to a decrease in leiomyoma size [16].

In our study cervical leiomyomas, which can affect mode of delivery, had the highest rate of increase in size compared to leiomyomas in other locations. Data regarding cervical leiomyomas is limited. Tian et al. described a series of 17 women with cervical leiomyoma during pregnancy. Sixteen of those women were delivered by cesarean delivery, mostly due to obstructed labor, and three underwent hysterectomy at the time of the cesarean section or shortly after due to post-operative complications. A statistically significant correlation was found between blood loss volume and leiomyoma size [17]. These data suggest an association between large cervical leiomyomas and adverse outcomes during delivery but clearly this is a topic in need of further investigation.

Besides its retrospective nature, this study has certain limitations. Information regarding ethnicity was unavailable. Size of uterine leiomyomas was estimated using area as opposed to volume. This was due to lack of data regarding a third dimension in most of the US exams performed. US exams were performed by different ultrasound technicians

Table 3 Correlation ( $r$ ) between basic parameters and the percent of change in size of the leiomyomas

\begin{tabular}{llllll}
\hline & \multicolumn{2}{l}{ Overall change in area } & & & Weekly change in area \\
\cline { 2 - 3 } \cline { 5 - 6 } & 1st to 2nd trimester & 2nd to 3rd trimester & & 1st to 2nd trimester & 2nd to 3rd trimester \\
\hline Age & -0.063 & -0.143 & -0.086 & -0.155 \\
Gravidity & -0.066 & -0.034 & -0.091 & -0.065 \\
Parity & -0.101 & -0.008 & -0.14 & -0.041 \\
BMl & 0.025 & -0.022 & 0.01 & -0.072 \\
No. of leiomyomas & -0.07 & 0.149 & -0.06 & ${ }^{0} 0.219$ \\
Pre-gestational size & -0.15 & -0.254 & -0.227 & -0.224 \\
Size at end of puerperium & 0.039 & -0.234 & -0.048 & -0.275
\end{tabular}

${ }^{a} P$ value $=0.024$. However, this correlation did not remain significant after adjusting for multiple comparisons 
as well as by physicians, possibly leading to operator bias. However, as all exams were performed by the same health provider, the same protocol was used for all exams. Uterine leiomyomas were measured during different gestational weeks. This was dealt by adjusting the results per week of gestation and calculating growth per week of gestation. The number of women included in our study constitutes one of the largest series presented to date on this topic. In the future, well planned prosepective studies focusing on women diagnosed with uterine leiomyomas during pregnancy should be able to address most of these limitations.

\section{Conclusions}

The main finding of our study is that uterine leiomyomas grow substantially during the 1st trimester of pregnancy but this trend is attenuated later in pregnancy with minimal growth towards the end of gestation. Further and prospective studies are needed to characterize change in leiomyomas during pregnancy and their clinical importance.

\section{Abbreviations}

BMI: Body mass index; HCG: Human chorionic gonadotropin; US: Ultrasound

\section{Acknowledgments}

Not applicable.

\section{Authors' contributions}

HHC: Data analysis, manuscript writing and editing. GK: Data analysis, manuscript writing and editing. TR: Data collection and management, manuscript editing. UD: Data analysis, manuscript editing. OT: Data collection and management, manuscript editing. AS: Project design, data analysis, manuscript editing. All authors have read and have approved the final version of the manuscript.

\section{Funding}

No funding was received for this study.

\section{Availability of data and materials}

The datasets used and/or analyzed during the current study are available from the corresponding author on reasonable request.

\section{Ethics approval and consent to participate}

The study was approved by the institutional ethical review board of Maccabi Health Services, IRB\# 38/2016. No informed consent was required.

\section{Consent for publication}

Not applicable.

\section{Competing interests}

The authors declare that they have no competing interests.

\section{Author details}

${ }^{1}$ Department of Obstetrics and Gynecology, Hadassah Hebrew University Medical Center, Ein-Kerem, PO Box 12000, 91120 Jerusalem, Israel.

${ }^{2}$ Maccabi Health Services, Jerusalem, Israel.

Received: 5 June 2019 Accepted: 12 July 2019

Published online: 22 July 2019

\section{References}

1. Tamaya T, Fujimoto J, Okada H. Comparison of cellular levels of steroid receptors in uterine leiomyoma and myometrium. Acta Obstet Gynecol Scand. 1985;64(4):307-9.

2. Maruo T, Ohara N, Wang J, Matsuo H. Sex steroidal regulation of uterine leiomyoma growth and apoptosis. Hum Reprod Update. 2004;10(3):207-20.
3. Stewart EA. Uterine fibroids. Lancet. 2001;357(9252):293-8.

4. Wallach EE, Vlahos NF. Uterine myomas: an overview of development clinical features, and management. Obstet Gynecol. 2004;104(2):393-406.

5. Sarais V, Cermisoni GC, Shimberni M, Alteri A, Papaleo E, Somigliana E, et al. Human chorionic gonadotropin as a possible mediator of leiomyoma growth during pregnancy: molecular mechanisms. Int J Mol Sci. 2017;18:9. https://doi.org/10.3390/ijms18092014

6. Ziecik AJ, Kaczmarek MM, Blitek A, Kowalczyk AE, Li X, Rahman NA. Novel biological and possible applicable roles of $\mathrm{LH} / \mathrm{hCG}$ receptor. Mol Cell Endocrinol. 2007;269:51-60.

7. De Vivo A, Mancuso A, Giacobbe A, Maggio Savasta L, De Dominici R, Dugo $\mathrm{N}$, et al. Uterine myomas during pregnancy: a longitudinal sonographic study. Ultrasound Obstet Gynecol. 2011;37(3):361-5.

8. Qidwai Gl, Caughey AB, Jacoby AF. Obstetric outcomes in women with sonographically identified uterine leiomyomata. Obstet Gynecol. 2006; 107(2, Part 1):376-82

9. Laughlin SK, Baird DD, Savitz DA, Herring AH, Hartmann KE. Prevalence of uterine leiomyomas in the first trimester of pregnancy: an ultrasound screening study. Obstet Gynecol. 2009;113(3):630.

10. Exacoustos C, Rosati P. Ultrasound diagnosis of uterine myomas and complications in pregnancy. Obstet Gynecol. 1993;82(1):97-101.

11. Katz VL, Dotters DJ, Droegemueller W. Complications of uterine leiomyomas in pregnancy. Obstet Gynecol. 1989;73(4):593-6.

12. Vitagliano A, Noventa M, Di Spiezio Sardo A, et al. Uterine fibroid size modifications during pregnancy and puerperium: evidence from the first systematic review of literature. Arch Gynecol Obstet. 2018;297(4):823-35.

13. Aharoni A, Reiter A, Golan D, Paltiely Y, Sharf M. Patterns of growth of uterine leiomyomas during pregnancy. A prospective longitudinal study. $\mathrm{Br}$ J Obstet Gynaecol. 1988;95(5):510-3.

14. Neiger R, Sonek JD, Croom CS, Ventolini G. Pregnancy-related changes in the size of uterine leiomyomas. J Reprod Med. 2006;51(9):671-4.

15. Lev-Toaff ANNAS, et al. Leiomyomas in pregnancy: sonographic study. Radiology. 1987;164(2):375-80.

16. Ciavattini A, Delli Carpini G, Clemente N, Moriconi L, Gentili C, Di Giuseppe J. Growth trend of small uterine fibroids and human chorionic gonadotropin serum levels in early pregnancy: an observational study. Fertil Steril. 2016:105(5):1255-60.

17. Tian J, Hu W. Cervical leiomyomas in prengnancy: report of 17 cases. Aust N Z J Obstet Gynaecol. 2012;52(3):258-61.

\section{Publisher's Note}

Springer Nature remains neutral with regard to jurisdictional claims in published maps and institutional affiliations.
Ready to submit your research? Choose BMC and benefit from:
- fast, convenient online submission
- thorough peer review by experienced researchers in your field
- rapid publication on acceptance
- support for research data, including large and complex data types
- gold Open Access which fosters wider collaboration and increased citations
- maximum visibility for your research: over $100 \mathrm{M}$ website views per year
At $\mathrm{BMC}$, research is always in progress.
Learn more biomedcentral.com/submissions 